\title{
IT'S ONLY A CUT FINGER
}

\author{
RoberT H. C. Robins, M.B., B.ChIR., F.R.C.S. \\ Orthopadic Surgeon, Royal Cornwall Infirmary, Truro; Hunterian Professor, Royal College of Surgeons
}

MINOR injuries are of two sorts: those which are considered trivial by the patient and those which are liable to be underestimated by the doctor (Fig. I). The former are indeed minor in all respects: abrasions, tiny lacerations and penetrating wounds, the importance of which lies in the danger of infection. The others embrace all those injuries not requiring admission to hospital and often thought unworthy of the attention of a consultant. Yet the penalties of failure can be severe, even after injury to a single digit, if infection, œdema or disuse should cripple the rest of the hand (Fig. 2); the potentialities for this exist wherever the surgeon fails to distinguish between an incised wound and one caused by crushing (Fig. 3). In this article I shall discuss the management of patients whose treatment can be performed without the need for admission to hospital, but assuming that the proper facilities are available, including anæsthesia, asepsis and instruments appropriate for hand surgery (Fig. 4).

\section{Prevention of Infection}

Sepsis rarely follows severe hand injuries which receive attention in a main operating theatre and in which the wound gets the treatment it deserves. Infections typically complicate trivial wounds and result mainly from their neglect. By their nature, these injuries seldom come to hospital but, as doctors, it is our duty to educate the public and, more particularly, nurses and first-aid workers in factories. Common sense and cleanliness are the main requirements. Common sense implies the ability to distinguish a penetrating wound or one which requires suturing from a superficial cut or abrasion. The former needs hospital treatment, while the latter requires thorough cleansing and. the application of a simple sterile dressing. Antiseptics should be avoided. The removal of splinters and foreign bodies must be complete and if a piece is retained the patient should be referred to hospital. The question of tetanus prophylaxis is appropriate here. The value of serum is in doubt. I am of the opinion that all attention should be paid to the wound and, if there is reason to question its contamination, that an antibiotic should be given and serum withheld. Patients who have been immunized should receive a

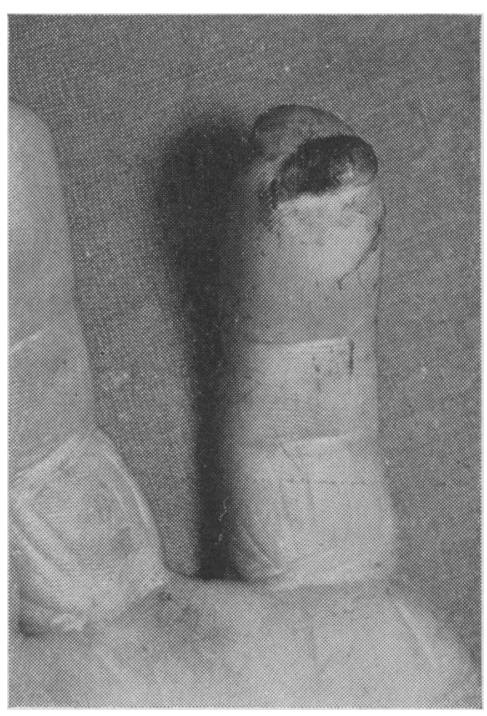

FIG. I.-Granulations growing from an untreated incised wound.

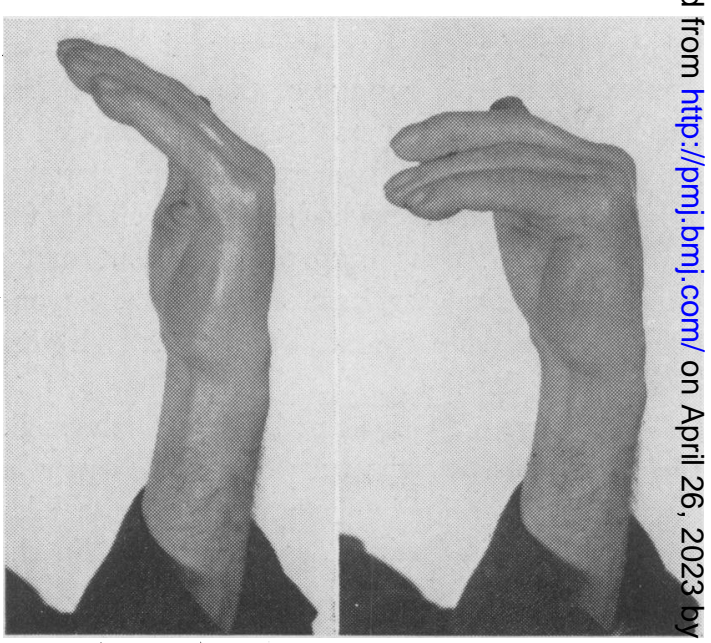

FIG. 2.-Finger stiffness following hæmatoma ard infection.

booster dose of toxoid, and those who have ngt should be given their first, not with the idea of immediate protection, but in order to start $\frac{\mathbb{Q}^{a}}{2}$ 


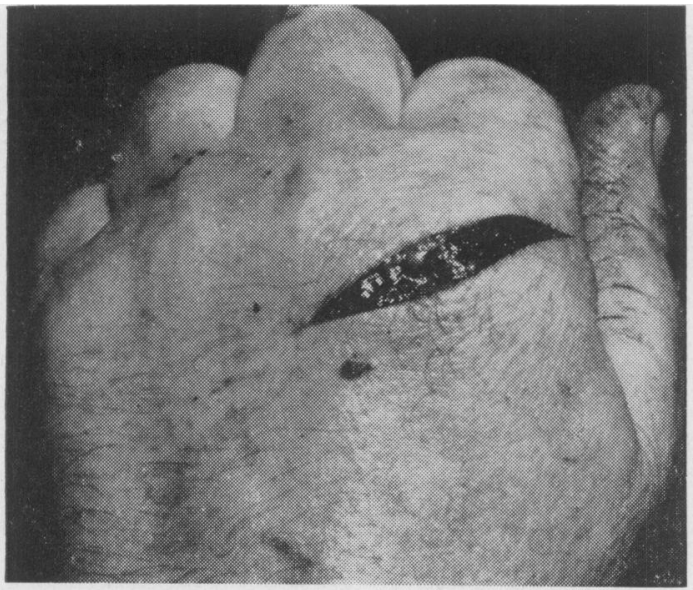

(a)

FIG. 3.- (a) Incised wound. (b) Lacerated wound wit' crushing of the margins.

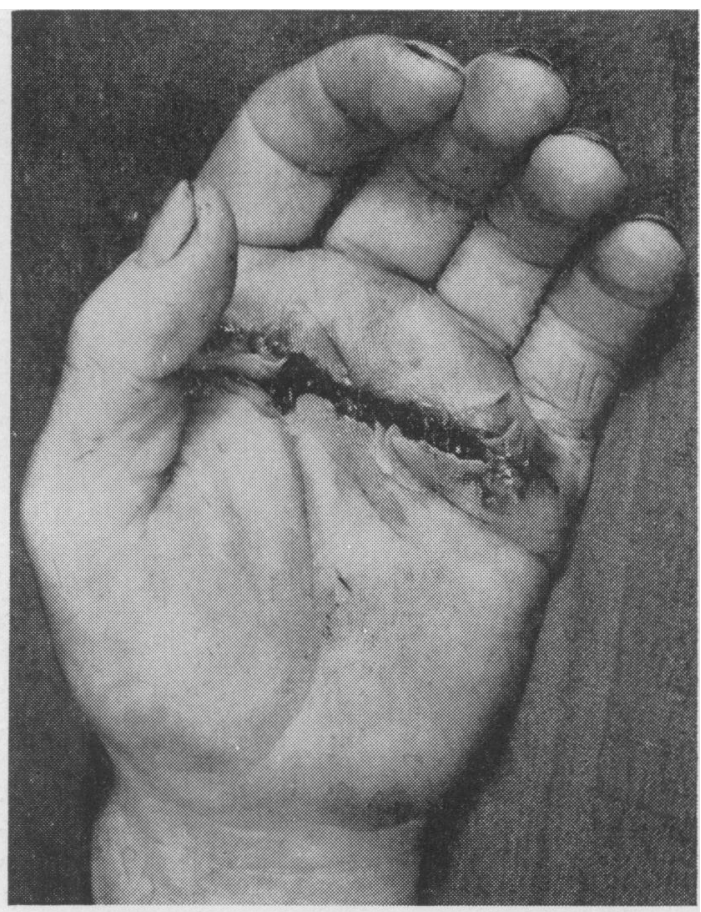

(b)

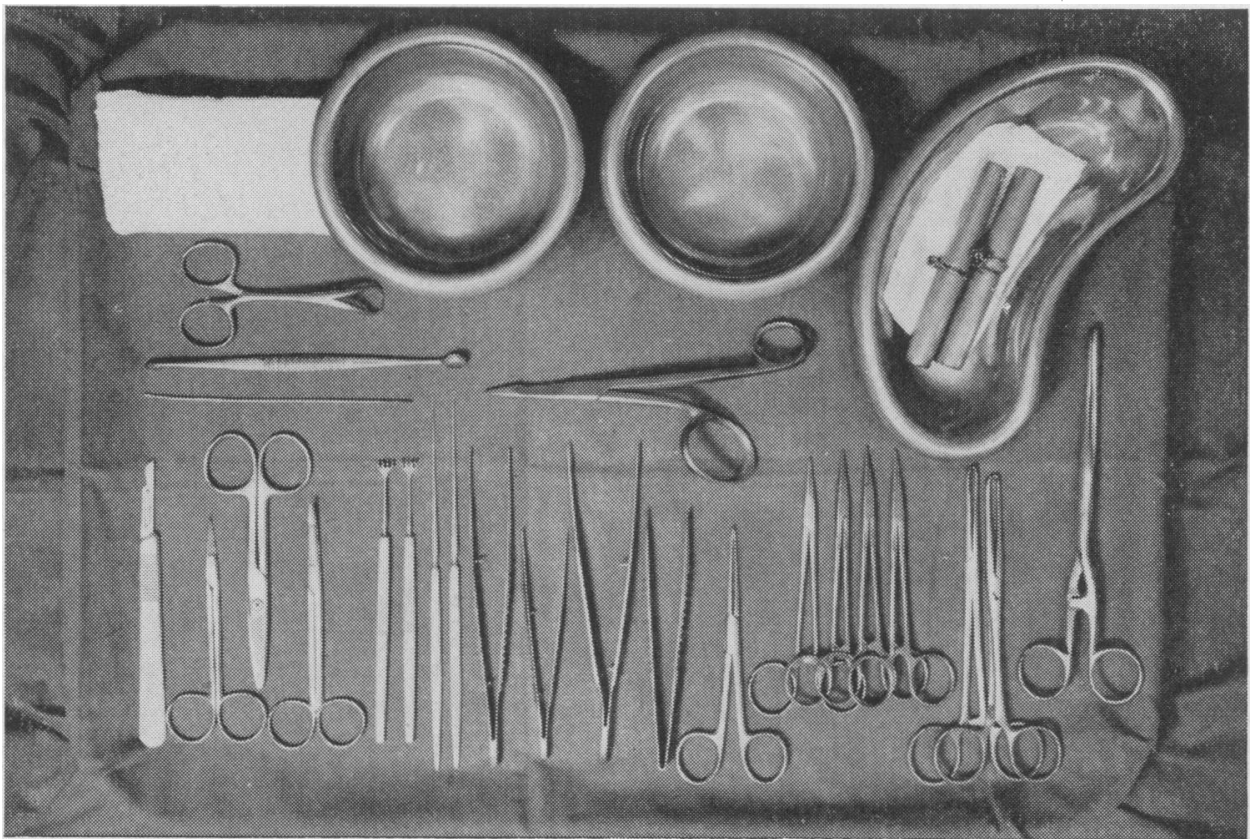

FIG. 4.-Routine set for minor hand surgery.

programme of active immunization. Leaving aside the possibility of tetanus, full antibiotic cover should be given to all patients with signifi- cant wounds which have been untreated for more than 6 hours, especially those caused by crushing. Patients with crush injuries and lesions for which 
special treatment has been necessary, such as tendon repair or skin grafting, should receive one large dose of crystalline penicillin by injection. Clean incised wounds of skin and subcutaneous tissue need no antibiotic at all, provided that they have had the proper local treatment.

\section{Principles of Wound Treatment}

The first need is to ask the patient the cause of the injury, when it occurred and what has happened to the wound since. Wounds are caused by the cutting, tearing or crushing of tissues and these distinctions apply as much to small injuries of the fingers as to mutilating conditions. Incised wounds may be expected to heal with minimal reaction and thus permit the suture of a tendon or digital nerve if there is no other contraindication. Contused wounds have been partially devitalized; at best they are liable to delayed healing with accompanying œdema and fibrosis, and at worst to actual sloughing of skin and subcutaneous tissue. In such circumstances repair of deep structures is not only contraindicated but may be harmful. The time between accident and definitive treatment influences the risk of infection and delay in healing, as does the application of strong chemicals or meddlesome interference with the wound.

The choice of anæsthetic depends on the circumstances. Most of the procedures I shall describe can be performed under digital nerve block, using $\mathrm{r} \%$ Xylocaine solution; all can be done under brachial plexus block which allows the use of a pneumatic tourniquet. General anæsthesia is often more convenient, particularly in operations for skin replacement. A bloodless field can be obtained adequately by elevation of the arm and application of a pneumatic cuff. It should be used whenever wound exploration is necessary or any deep structure is to be repaired, but not for skin grafting in any form.

Dressings should be simple. Fluffed dry gauze is the most generally applicable, and petroleum jelly gauze next to the wound if adherence is likely to be a problem. A light compression bandage may be used and a protective plaster slab if it is necessary to control the position of a part of the hand. Individual finger dressings with stockinette applied on a frame are very convenient, but care must be taken to avoid a constricting turn at the base of the digit. For the first few days only, elevation in a sling may be desirable, but from the beginning the patient must be instructed in exercises for the shoulder and uninjured parts of the hand, and it must be clear that he understands them.

\section{Superficial Wounds Without Skin Loss}

Clean incised wounds in the fingers do not need to be excised. Simple cleansing is necessary and the wound inspected for damage to deep structures. The skin is then sutured with fine monofilament nylon or other suitable material and a simple drye dressing applied.

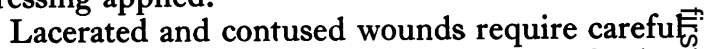
handling in order to avoid inflicting furthe damage. Excision, in the true sense of the word $\frac{C}{0}$ is unnecessary but devitalized tissue and foreign material must be removed. Skin closure should bee as precise as possible, provided that there is no tension. Where a flap of skin is based distally, if? should be thinned by removal of fatty tissue beforẹ suture.

\section{Superficial Wounds With Skin Loss}

Loss of skin demands immediate replacement by grafting. The special problem of amputation. of the tip of the finger will be considered later Elsewhere in the hand, and notably on the sides of the digits, small areas of skin loss are adequately treated by free split skin grafts. These majo conveniently be taken from the volar aspect of the forearm after the donor area has been demarcated. by infiltration with local analgesic. (For cosmetie reasons, women should be offered the alternative of the thigh). It is always wise to suture the graft in its position. Five days after operation is reasonable time to perform the first dressing, tut there are occasions when it should be done soofiro particularly if there is a chance of hæmatomas while in other instances it may be left for a week o more.

\section{Injuries Around the Nail}

Subungual hæmatoma is a painful condition easily relieved by trephining the nail for its: release. Contact with the red-hot end of a paper clip is effective. Radiographs should be taken exclude a fracture of the terminal phalanx; though this will not affect the treatment.

More extensive injuries are usually caused b\$․․ crushing and if the nail fold is crushed, soms deformity of the nail is likely to ensue. There are no special features about treatment except that the nail should not be sacrificed needlessl because it acts as a natural protection during the healing phase, even if shed later. If the nail dislocated, however, there is little virtue io preserving it and it should be removed at the outset.

\section{Penetrating Wounds and Foreign Bodies}

The history here is important. Penetrating wounds caused by inorganic materials, such as glass and metal, may be treated conservativety unless they are contaminated or a deep structure has been divided. In contrast, wounds caused 
organic materials, such as wooden splinters or bones, should be explored. The same principle applies to foreign bodies. Organic foreign bodies must not be allowed to remain because of the risk of infection, including tetanus. By contrast glass, fragments of needles or air gun pellets may often be left for elective removal later. Foreign bodies in the hand may be very difficult to find and casualty officers are liable to embark on their search with insufficient forethought and preparation. In the case of radio-opaque objects, films should be taken in two planes. Glass will be visible only if it contains lead.

\section{Amputations of The Finger Tip}

If the result of treatment of a traumatic amputation is a painful and tender end to the finger or an unstable scar, the method chosen has failed. The point which I mean to emphasize is this:Length is important, but the quality of the stump is more so. The typical problem is loss of skin and a variable amount of pulp, the level of amputation passing through the terminal phalanx or the pulp distal to it. If the direction is oblique and in a dorsal direction, so that the main loss of tissue involves the nail and underlying structures, it is usually best to make a formal amputation through the distal phalanx removing nail bed and fold (Fig. 5). The volar skin flap will then give ideal cover; being the proper local skin and having normal sensibility, it will stand up to the stresses of hard manual work. Such a procedure is advisable in all instances when local skin flaps can be fashioned without undue shortening of the digit. It may also be best for unskilled labourers even when significant shortening is needed to get satisfactory closure, and especially in elderly patients where rapid healing is important to offset the danger of finger stiffness.

This will leave a large group of cases which need skin cover by grafting. A split skin graft seldom gives satisfactory permanent cover to the finger tip unless the defect is superficial and likely to be protected by the new nail as it grows. It is, however, a valuable technique as a temporary expedient in, for instance, a patient who comes late for treatment after an injury at sea; definitive surgery can then be deferred until primary healing has been achieved and the danger of sepsis passed.

The usual alternatives are a full thickness graft or a flap from elsewhere in the hand. Neither of these will develop more than protective sensibility, but a flap gives a more handsome cosmeic result and better durability. Against a flap are the facts that it means two operations, a longer disability period, admission to hospital for a few days if the patient does not live near enough to attend daily, and a decided risk of finger stiffness if the operation

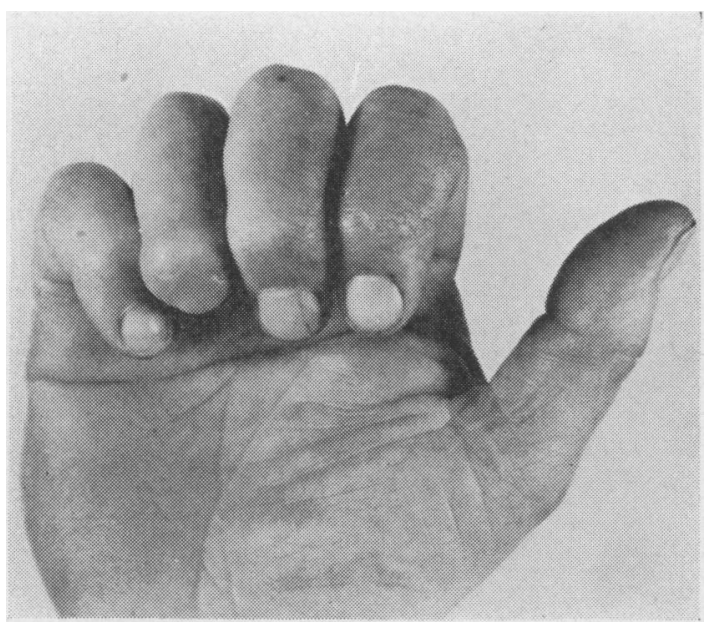

Fig. 5.-Amputation through the terminal segment of the ring finger. The digit is shown in full flexion; the scar is away from the important tactile area.

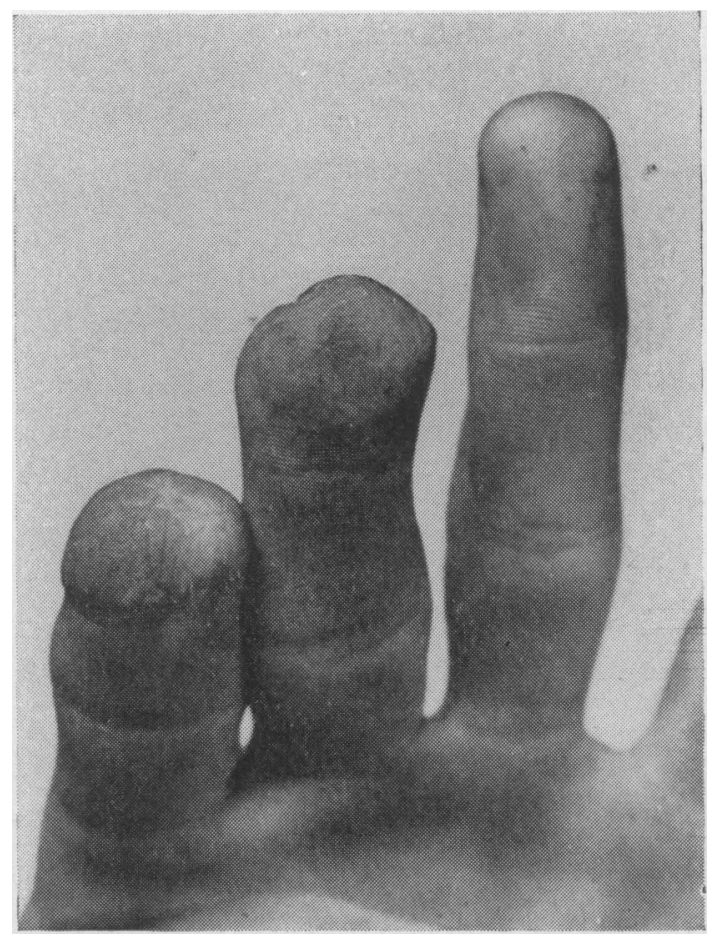

Fig. 6.-Two fingers treated by full thickness grafts.

is not skilfully performed or the selection of patient has been misjudged. An advantage of a free graft is its tendency to contract and therefore to pull towards itself the marginal and normal skin of the volar pad to which it was sutured; thus the final grafted area several months later will be smaller than the original defect. Moreover it 
sometimes happens that sufficient local skin is available to cover the exposed bone and a free graft will then close what remains.

The technique of full thickness skin grafting is first to prepare the affected finger, to take a pattern of the defect cut in glove rubber or sterile foil, then to map this out on the donor area, cut the graft and suture it to the defect. In preparing the finger, minimal excision is performed, removing only such tissue as is contaminated or devitalized. Hæmostasis must be meticulous and can be achieved by pressure with hot moist packs. While waiting for the field to become dry the graft is taken. The most convenient donor area is the bend of the elbow, because after removing the graft, the defect can be closed by transverse suture in the line of the skin crease. In cutting the graft, the skin is lifted at its margin with a fine hook and great care taken to remove every trace of fat from its under-surface. Fixation to the end of the finger is important and fine atraumatic sutures should be used. Four can be left long enough to tie a small pressure dressing on top. The graft is inspected after five days and thereafter as necessary. The period off work for a manual worker is likely to be 6 to 8 weeks (Fig. 6).

For selected patients, a skin flap provides the best method of repair. The donor site may be the thenar eminence, adjacent digit (cross-finger flap) or, in the case of the thumb, the radial side of the proximal segment of the index finger. In raising a thenar flap (Fig. 7) it is important to judge the correct site accurately by flexing the affected finger in its natural direction and choosing the area of skin which comes into contact with the amputated tip. In cutting the flap some subcutaneous tissue is usually left behind as a bed for a small free graft (either split skin or full thickness) which is sutured to the edges of the defect. The thenar flap is sutured to the margins of the amputation wound and a small dressing interposed between the bridge of the flap and the free graft beneath. Strapping or a plaster cast should be used to maintain the finger in its correct position and to prevent too much tension on the flap. Dressings require changing about every fourth day and the flap may be detached after two weeks; healing should be complete in another fortnight. Failure-loss of the graft, painful donor area or finger stiffness-may be due to wrong selection of patients, incorrect siting of the flap, positioning of the finger too flexed or too extended (the flap should be at right angles to the thenar skin), or detachment too early or too late.

The principle of a cross-finger flap is exactly the same. The donor area is the lateral aspect and dorsum of the middle segment of an adjacent finger. It must be large enough to cover the defect and still allow for a short bridge and its length

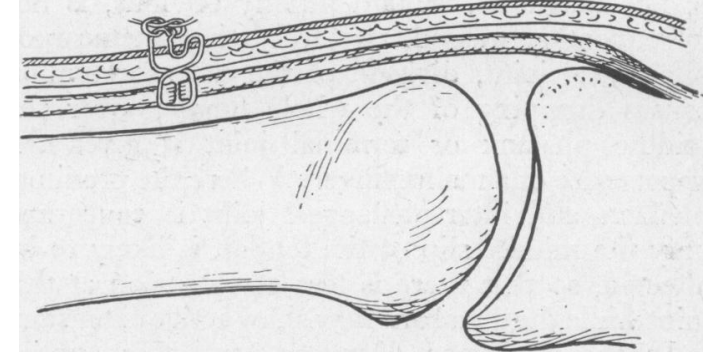

FIG. 8.-Figure-of-eight suture. (After Bunnell).

should not exceed one and one-third times its breadth. On no account may its base extend in front of the mid-lateral line, and a covering of paratenon must be left on the extensor tendon. The flap is rotated through 180 degrees in order to suture it to the defect; split skin graft covers the donor area and extends on to the bridge between the two fingers. A plaster cast is used to maintain the position and a window is cut in it to observe the vascularity of the flap which should be ready for detachment after a fortnight.

\section{Digital Nerve Injuries}

Examination for sensory loss is essential in all patients with injuries of the hand. A divided digital nerve in an incised wound is suited to primary repair, though some extension of the wound may be necessary. Two fine atraumatic sutures are passed through the perineurium, care being taken to ensure that the ends of the nerve are in their correct rotational relationship. After closure of the skin the finger is splinted in mid flexion to relieve the suture line of tension.

In contused and contaminated wounds, no sutures should be inserted but it is worth laying the divided nerve with its ends approximated, because a reasonable degree of natural recovery sometimes ensues.

\section{Flexor Tendon Injuries}

These should not be submitted to surgical repair as an out-patient. If, on exploration of a wound, the flexor tendon is found to be intact but its sheath to be open, penicillin solution should be instilled into its lumen.

\section{Extensor Tendon Injuries}

Extensor tendons may be considered for primary repair in clean, incised wounds. Admission to hospital is not necessary if the lesion is situated at a level where the proximal end is not likely to have retracted far and therefore can be found without much extension of the wound of injury. In practice, this means from the metacarpal heads distally. 
The diagnosis, though usually obvious, is not infrequently missed. Over the metacarpophalangeal joint, division of the extensor tendon causes dropping of the whole finger; over the middle phalanx or terminal joint it gives the appearance of a mallet finger. Where the proximal phalanx and inter-phalangeal joint is concerned only the middle slip of the tendon is likely to be affected, so that there is loss of extension at this joint while the distal one may show active extension.

In the situations discussed, and this applies also if a joint has been opened, a figure-of-eight wire suture is generally applicable, because retraction is not a serious problem and the tendon is approaching flat in section. Moreover, there is little subcutaneous tissue to act as a cushion and the absence of buried suture material is an important advantage.

The technique is simple. The tendon and skin are incorporated in a figure-of-eight manner (Fig. 8), so that the ends of the former are auto- matically approximated as the suture is tieds: Three sutures are usually needed and they should all be inserted before the first is tied. They arez left in position for three weeks, during which time ${ }_{\Omega}^{\mathbb{\mathbb { D }}}$ the digit is splinted in the extended position.

\section{Conclusion}

All these operations require precise technique, $\frac{\bar{C}}{0}$ and if they are applied without due regard for $\overline{\bar{c}}$. basic principles of asepsis and surgical skill they $\widehat{\nabla}$ will bring disrepute. I do not suggest that they are suited to indiscriminate use in casualty depart- $\infty$ ments, but I do believe that this is the standard $\vec{O}$ which should be aimed at in out-patient accident $\overrightarrow{\vec{\omega}}$ work, and if that is achieved the treatment of these $\omega$ injuries will contribute to an ideal basic surgicalo training.

Figures $1,2,3,4,5,6$ and 8 are reproduced by permission from 'Injuries and Infections of the Hand' (Edward Arnold, London). 